\title{
Ingénieries entre recherche et formation
}

Élèves-professeurs en mathématiques aux prises avec des ingénieries didactiques issues de la recherche. Un dispositif de formation à portée phénoménotechnique

A phenomenotechnical training device

\section{Maggy Schneider et Pierre Job}

\section{OpenEdition}

\section{Journals}

Édition électronique

URL : https://journals.openedition.org/educationdidactique/2508

DOI : 10.4000/educationdidactique.2508

ISSN : 2111-4838

\section{Éditeur}

Presses universitaires de Rennes

\section{Édition imprimée}

Date de publication : 8 juillet 2016

Pagination : $91-112$

ISBN : 9782753551787

ISSN : 1956-3485

\section{Référence électronique}

Maggy Schneider et Pierre Job, « Ingénieries entre recherche et formation », Éducation et didactique [En ligne], 10-2 | 2016, mis en ligne le 08 juillet 2018, consulté le 16 septembre 2022. URL : http:// journals.openedition.org/educationdidactique/2508; DOI : https://doi.org/10.4000/ educationdidactique. 2508 


\title{
INGÉNIERIES ENTRE RECHERCHE ET FORMATION ÉLÈVES-PROFESSEURS EN MATHÉMATIQUES AUX PRISES AVEC DES INGÉNIERIES DIDACTIQUES ISSUES DE LA RECHERCHE. UN DISPOSITIF DE FORMATION À PORTÉE PHÉNOMÉNOTECHNIQUE
}

\author{
Maggy Schneider \\ Université de Liège, Laboratoire de didactique des mathématiques \\ Pierre Job, Université de Liège \\ Avec la collaboration de Hilda Rosseel, que nous tenons à remercier.
}

\begin{abstract}
La question de recherche traitée dans cet article consiste à identifier le type d'indicateurs d'une aptitude requise par le métier d'enseignant de mathématiques : savoir analyser des situations didactiques, au moyen de concepts épistémologiques et didactiques, au-delà du seul discours sur les valeurs alimentées par des idéologies non questionnées, en particulier celles inspirées du paradigme socio-constructiviste.

Elle a été traitée dans le cadre d'une formation initiale d'élèves-professeurs de lycée qui a ainsi joué un rôle phénoménotechnique par le biais d'un dispositif de comparaison d'ingénieries issues de la recherche en didactique et portant sur le concept de dérivée. L'observation est relative à l'analyse mathématique et aux praxéologies " modélisation » qui y préparent des élèves du secondaire en vue de leur faire dépasser une vision empiriste des grandeurs géométriques et physiques modélisées.

Larticle montre que l'identification des indicateurs recherchés peut difficilement faire l'économie d'un double niveau d'analyses a priori : d'abord, celles qui portent sur les situations didactiques qu'on fait travailler aux élèvesprofesseurs; ensuite, à la lumière de ce premier niveau, des analyses relatives à la manière dont ceux-ci s'emparent des spécificités didactiques du même matériau pour mener une réflexion qui leur est propre.
\end{abstract}

Mots-clés : formation initiale d'élèves-professeurs de lycée, idéologies d'enseignement, indicateurs d'entrée dans l'analyse didactique, dispositif de formation à portée phénoménotechnique, ingénieries didactiques.

\section{A phenomenotechnical training device}

In this paper we study the question of identifying indicators of a specific skill required for teaching mathematics: being able to analyze didactic situations using epistemological and didactic concepts and not just arguments based on values drawn from unquestioned ideologies, notably those inspired by socio-constructivist theories.

This question was addressed in the framework of an initial teacher training. This training played a phenomenotechnical role thanks to a tool allowing comparisons of didactic engineering devised for the needs of researches on the derivative concept. The data relate to mathematical analysis and modelling praxeologies whose aim is to help secondary school students to overcome an empirical understanding of geometrical and physical magnitudes.

The paper shows that the identification of the sought after indicators can hardly bypass a two level prior analysis. The first level pertains to the didactic engineering the student teachers have to work on. The second level, relying on his first one, highlights the features of these didactic engineering the student teachers use to conduct their own thinking.

Keywords: high school initial teacher training, teaching ideologies, acquisition of didactic analysis skills indicators, phenomenotechnical training device, didactic engineering. 


\section{INTRODUCTION}

La question de recherche traitée ici est relative à l'exploitation d'ingénieries didactiques issues de la recherche dans le cadre d'une formation initiale d'élèves-professeurs.

Il ne s'agit pas ici d'évaluer les effets de cette formation mais d'en montrer la portée phénoménotechnique par la mise en évidence d'indicateurs caractérisant la manière dont des élèves-professeurs s'approprient des dispositifs inspirés du paradigme socio-constructiviste. Le mot "phénoménotechnique » est à prendre au sens de la didactique des mathématiques dite fondamentale, développée en France suite aux travaux de Brousseau (1996): les ingénieries didactiques sont avant tout des méthodologies de recherche supposées créer des observables qui deviennent "phénomènes " à la lumière de théories didactiques. Ce qui n'empêche pas qu'elles servent aussi de dispositifs d'enseignement : un « avantage collatéral » en quelque sorte. Ce point de vue phénoménotechnique est développé plus amplement par Artigue (1990) à propos des ingénieries et par Schneider (sous presse) en ce qui concerne les modèles épistémologiques de référence des chercheurs. Cet article illustre ce double point de vue au niveau de la formation des enseignants: une ingénierie didactique y est exploitée à la fois comme moyen de formation et comme dispositif de recherche sur les difficultés d'apprentissage des professeurs en formation dans leur appropriation de certains dispositifs didactiques.

Mais la formation dans laquelle s'intègre notre dispositif de recherche vise des objectifs qui relèvent, eux, d'un choix de valeurs forcément sujet à débat. Si ce n'est pas principalement un tel questionnement que nous voulons soulever ici, nous nous devons malgré tout d'afficher ces choix que nous avons faits en tant que formateurs et leurs raisons d'être car c'est ce qui permettra au lecteur de pouvoir situer par luimême les observables et l'analyse que nous en proposons. C'est ce que nous faisons à la section 1 en nous situant comme formateurs. En même temps, nous décrirons aussi le contexte dans lequel s'est déroulé la récolte des données ainsi que plusieurs concepts qui serviront à les analyser. Nous pourrons ainsi situer et préciser notre question de recherche, à la section 2 , en troquant notre casquette de formateurs pour celui de chercheurs.

\section{UN CADRAGE THÉORIQUE PERMETTANT DE CARACTÉRISER UN CONTEXTE DE FORMATION DANS LEQUEL S'INTÈGRE LA QUESTION DE RECHERCHE ET DE LA TRAITER}

\author{
Des « mathématiques comme problème \\ professionnel » au pedagogical content \\ knowledge
}

Un premier élément sur lequel notre formation prend appui tient dans une expression qu'explique et développe Cirade (2006), à savoir « les mathématiques comme problème professionnel $»$. Ce point de vue, d'autres chercheurs l'ont décrit et analysé selon des déclinaisons diverses. Commençons par la modélisation que fait Margolinas (2004) du milieu du professeur constitué de couches imbriquées les unes dans les autres: le niveau +3 des valeurs et conceptions sur l'enseignement / apprentissage fait de croyances, par exemple sur les vertus de l'activité de l'élève ; le niveau +2 de la construction didactique globale du thème mathématique dans lequel s'inscrit la séance : notions à étudier et apprentissages à réaliser ; le niveau +1 du projet didactique spécifique pour la séance observée : objectifs, planification du travail ; le niveau 0 de la réalisation de la séance, des interactions avec les élèves et de la prise de décision dans l'action; et le niveau -1 de l'observation de l'activité des élèves et de la régulation de leur travail. Par rapport à ce modèle, cette chercheuse montre la difficulté des élèves-professeurs (que nous appellerons indifféremment « stagiaires », " futurs enseignants », « étudiants » ou « formés ») à travailler sur le thème mathématique en considérant des aspects mathématiques et épistémologiques d'une manière un tant soit peu détachée des autres niveaux, se contentant souvent d'une réflexion portant sur des niveaux plus locaux, comme la gestion de la classe, tout en justifiant leurs choix didactiques par des considérations relatives aux valeurs. Ce qui ne signifie pas qu'il faille minimiser le caractère systémique nécessaire de toute réflexion relative à une préparation de cours devant intégrer tous les niveaux mais l'absence d'une analyse a priori au niveau du thème mathématique peut conduire à une réduction du champ de conscience de l'ensemble des possibles soit de l'action des élèves, soit de celle du professeur, le discours idéologique sur les valeurs servant alors de refuge pour justifier les choix faits. C'est l'analyse descendante centrée sur l'activité du professeur P : de P-noosphérien à 
P-observateur dans la classe qui nous intéresse ici en ce sens que nous nous sommes surtout polarisés sur des difficultés éprouvées par les élèves-professeurs en amont de leur activité en classe. Nous y reviendrons dans l'analyse.

De son côté, Bloch (1999) épingle les difficultés éprouvées par le professeur pour gérer ses interactions avec les élèves aux niveaux adidactiques du modèle de Margolinas et les étudie en dégageant, par une analyse ascendante du milieu matériel au milieu de référence, « les caractéristiques du milieu du professeur $»$. Elle insiste sur les conceptions très formelles que les futurs enseignants ont des mathématiques et qui entraveraient leur capacité à mettre en scène le savoir mathématique sous forme de problèmes et propose un critère de pertinence mathématique pour analyser les interactions de l'enseignant avec ses élèves (Bloch, 2009).

L'analyse de Cirade (2006) ou de Artaud et Cirade (2008) corroborent celles de Margolinas et de Bloch tout en les étoffant d'éléments complémentaires. D'abord, si les élèves-professeurs ont beaucoup de peine à reproblématiser les savoirs enseignés à partir de leurs raisons d'être, c'est souvent par méconnaissance pure et simple de ces dernières, qu'elles soient ou non encore actuelles. Ensuite et en conséquence sans doute, ces étudiants en formation ne parviennent à mettre en œuvre le paradigme socioconstructiviste ${ }^{l}$ prôné par l'institution scolaire qu'en concevant des activités qui conduisent à des organisations mathématiques juxtaposées plutôt qu'articulées les unes aux autres, aucune réflexion préalable plus globale n'autorisant l'idée d'un parcours d'étude qui leur sert d'ossature. En outre, les élèves-professeurs sont bien souvent incapables de questionner, voire même de décrire les enjeux épistémologiques de ces mêmes activités, qu'ils les aient conçues euxmêmes ou empruntées dans les manuels : « rien, ici, ne semble problématique. La description est celle d'un univers évident, transparent, qui va de soi » (Cirade, 2006). En bref, on constate là la difficulté d'un travail proprement mathématique qui hypothèque les préparations de cours en ce sens que les enjeux d'apprentissage y sont mal identifiés.

S'y ajoute une difficulté, plus spécifique des élèves-professeurs ayant une formation universitaire en mathématique. Elle relève du double forgetting dont parlait le mathématicien Félix Klein (18491925) : il s'agit en effet « d'oublier » les mathématiques scolaires avant d'entrer à l'université où seules les théories déductives ont droit de cité, mais aussi d'oublier les mathématiques de l'université avant de « retourner à l'école». Cela afin de concevoir un discours rationnel sur les pratiques mathématiques qui peut être compris des élèves du secondaire sans pour autant être calqué du discours du mathématicien. Mais Rouy (2007) observe ces élèves-professeurs éprouver beaucoup de peine à «identifier la possibilité de travailler dans une rationalité autre que celle proposée par la théorie connue d'eux seuls ", au point d'adopter souvent un discours qui ne relève d'aucun niveau de rationalité, considérant l'institution universitaire comme un monde théorique et l'institution secondaire comme un monde technique, sans autre forme de rationalité possible. Or, il s'agit, par exemple, de pouvoir justifier « d'une manière élémentaire » que la dérivée de la fonction sinus est la fonction cosinus ou de définir un angle orienté « de manière intuitive » sans devoir recourir à la théorie mathématique. Nous reviendrons, dans l'analyse, aux travaux de Rouy qui se sont inscrits dans le même dispositif de formation.

Ces chercheuses concluent, de leurs observations respectives, que de telles questions révèlent un manque d'assurance des élèves-professeurs par rapport au savoir ainsi qu'une posture « décentrée » qui leur fait chercher les réponses à l'extérieur, dans des manuels par exemple, pour éviter de questionner leurs propres apprentissages mathématiques. Et ce pourrait être ce qui les empêche de reconstruire, à des fins didactiques et pour un niveau d'étude donné, une problématisation possible des savoirs enseignés.

Au-delà des connaissances proprement mathématiques, Shulman (1987) identifie ce qu'il appelle le pedagogical content knowledge (PCK) pour décrire les différents savoirs à usage didactique susceptibles d'outiller la profession d'enseignant dans un espace culturel donné. Depuis l'article princeps de Shulman, on dénombre des citations et des usages du content knowledge (CK) et du PCK très nombreux autant que diversifiés (entre autres le travail de Ma, 1999), ainsi que de multiples classifications associées (dont, par exemple, celle de Ball et al., 2008) pour caractériser les connaissances (ou compétences) utiles aux enseignants dans leur métier. Ce n'est pas utiliser l'une ou l'autre d'entre elles pour évaluer un public donné d'enseignants qui nous intéresse ici mais plutôt mettre à jour une capacité qu'on ne peut identifier qu'à partir d'un regard spécifiquement didactique tel qu'on peut l'entendre dans l'école française de didactique des 
mathématiques. Une enquête approfondie sur la notion de PCK a été menée par Silvy et al. (2013) qui illustrent abondamment leurs propos à plusieurs niveaux de scolarité. Leur investigation est publiée dans le volume 7(1) de la présente revue et nous y renvoyons le lecteur. Plus récemment, Clivaz (2014) fait l'état de la littérature sur ce sujet. Retenons des analyses des chercheurs précités une parenté entre le PCK de Shulman et ce que Brousseau (1998) nomme «l'épistémologie pratique des professeurs ». Car le plus important à nos yeux, dans le PCK, est la prise en compte systémique de deux domaines de connaissance jusque-là séparés : des connaissances liées au savoir à enseigner, d'une part, et des connaissances de type pédagogique, d'autre part :

It (PCK) represents the blending of content and pedagogy into an understanding of how particular topics, problems, or issues are organized, represented, and adapted to the diverse interests and abilities of learners, and presented for instruction. Pedagogical content knowledge is the category most likely to distinguish the understanding of the content specialist from that of the pedagogue (Shulman, 1987, p. 8, cité par Clivaz, 2004, p. 25).

Le concept de PCK est pour nous un idéal-type au sens du sociologue allemand Weber (cité par Bourdieu et al., 1983) soit, en substance, un concept aux contours tranchés, qui aide à situer des objets ou des phénomènes réels, mais qui a souvent besoin d'un complément de sens quand on l'utilise dans un contexte déterminé. S'il nous intéresse ici, c'est parce que, comme dit plus haut, il articule connaissances pédagogiques et connaissances disciplinaires. Cependant, cette articulation suppose, du point de vue de la didactique des mathématiques, un questionnement du savoir, de ses raisons d'être et de sa transposition didactique. Ce regard nous a amenés à identifier une spécification particulière du PCK qui constitue une pierre d'achoppement propre à la formation des futurs professeurs de mathématiques du lycée (niveau d'étude correspondant à la tranche d'âge 14-17 ans en Belgique). C'est l'objet de la section suivante.
Le contexte didactique de l'observation : les praxéologies « modélisation » nécessaires et non identifiées

Nous avons évoqué, à la section 1.0, la difficulté éprouvée par les élèves-professeurs à réfléchir leur enseignement au niveau du thème mathématique et de ses enjeux épistémologiques et cognitifs, difficulté liée tant à leurs apprentissages mathématiques qu'au pedagogical content knowledge. Cette difficulté prend une forme particulière dans le cas des formés qui se destinent à être professeurs de lycée en raison de la proximité de ce niveau d'enseignement avec l'université. Pour formaliser cela mais aussi pour fournir au lecteur des référents utiles pour apprécier l'analyse faite à la section 2, nous nous référerons aux notions de praxéologies «modélisation » et de praxéologies « déduction» (Schneider, 2008, 2011a) en les commentant largement par des éléments qui alimenteront précisément l'analyse faite plus loin.

Le concept de praxéologie est emprunté à Chevallard (1999), lequel l'utilise pour modéliser toute activité humaine et en particulier l'activité mathématique, qu'elle soit de l'ordre de la production ou de celle de l'utilisation. On peut expliquer brièvement ce concept à partir de son étymologie : un bloc «praxis » rassemble une tâche qu'on se donne et la technique exploitée pour la réaliser tandis que le bloc « logos» reprend un discours technologique ou théorique dont la fonction majeure est de justifier la technique utilisée et de la rendre intelligible en regard de la tâche visée. Pour décrire et analyser la difficulté à enseigner, qu'elle soit celle d'un élèveprofesseur ou celle d'un enseignant chevronné, nous pensons utile de distinguer deux types de praxéologies qui, faute d'être clairement identifiées, sont mêlées de manière inextricable au sein des cours dispensés dans le secondaire de sorte que ni l'une, ni l'autre n'ont de visibilité pour les élèves. Il s'agit de la distinction entre praxéologies « modélisation » et praxéologies « déduction » enseignée par Schneider (2008) à l'adresse des élèves-professeurs sur base de recherches publiées (Schneider, 1988 / 2011b).

Les praxéologies "modélisation " sont des praxéologies dans lesquelles les tâches consistent à modéliser par des concepts mathématiques des systèmes intra ou extra-mathématiques faits d'objets préconstruits au sens de Chevallard (1991). Ceuxci, telles les aires, les vitesses ou les tangentes, ne sont pas définis d'emblée par le biais de concepts 
mathématiques, en l'occurrence le concept de limite. Ils existent seulement par le truchement d'une désignation, non pas construits mais présentés « par une deixis qui est un appel à la complicité dans la reconnaissance ontologique » (ibid., p. 91). On cherche, dans un premier temps, non à les définir mais à les déterminer par l'une ou l'autre technique en s'appuyant sur les convictions, images, intuitions que l'on peut avoir à leur propos. Ils fonctionnent alors comme des "objets mentaux» au sens de Freudenthal (1973), soit en quelque sorte comme des substituts de concepts (Schneider, 1988). Et, dans cette perspective, les modes de validation se démarquent des preuves déductives en vigueur dans les théories axiomatisées: la validation peut être pragmatique en ce sens que la technique est " éprouvée » en la testant pour retrouver des résultats qui avaient déjà été établis d'une autre manière, ce qui a poussé Job et Schneider (2014) à parler de " praxéologies pragmatiques ». Ou encore, on s'autorise dans ces praxéologies à justifier les techniques et leurs résultats par des arguments intuitifs liés à des connaissances empiriques que l'on peut avoir sur les préconstruits, ici des intuitions liées aux aires ou vitesses comme, par exemple, l'idée d'un ordre sur les aires de surfaces emboîtées. Ce mode de justification est amplement illustré dans la deuxième partie de cet article. Ces praxéologies "modélisation » conduisent ensuite à des praxéologies « déduction » dans lesquelles les objets préconstruits laissent place à des concepts proprement mathématiques, existant cette fois par le truchement d'une définition qui va donner prise au raisonnement déductif. C'est qu'alors, les tâches majeures sont axées sur la constitution d'une organisation déductive : il s'agit, par exemple, d'établir un système d'axiomes "simple » et non redondant, de conjecturer un ordre d'agencement de théorèmes et de le faire aboutir sur base des règles d'inférence du calcul propositionnel et de celui des prédicats... Dans cette perspective, les aires sont définies comme des intégrales et les vitesses comme des dérivées, les unes et les autres étant donc des cas particuliers du concept de limite qui, formulé en termes de quantificateurs et d'inégalités, inspire alors un mode de preuves exemptes de toute considération géométrique ou cinématique.

L'intérêt des praxéologies «modélisation » dans un cursus scolaire en mathématiques réside dans les doutes qu'expriment les élèves quant à la pertinence de certains modèles mathématiques. Ainsi, une fois la vitesse instantanée définie comme une dérivée, des élèves prétendent qu'une telle vitesse n'existe pas, se référant à l'impossibilité de la déterminer exactement par des observations et des mesures: « le temps de chronométrer et du temps s'est déjà écoulé » ou encore " en un temps nul, aucun espace n'est parcouru » (Schneider, 1992). De même, ils doutent que la limite d'une suite d'aires de rectangles puisse égaler exactement une aire curviligne car «les rectangles n'épousent pas parfaitement la courbe » ou « se réduisent, à la limite, en segments » (Schneider, 1991a). Ou encore pensent la tangente comme «limite » de sécantes sans qu'aucune topologie n'ait été définie sur l'ensemble des droites plutôt que comme droite définie à partir de sa pente, c'està-dire par le biais d'une limite de fonction au sens mathématique (Schneider, 1991b). De telles réactions relèvent, ainsi que bien des erreurs que nous ne reprendrons pas ici, d'un positivisme empirique qui s'érige en obstacle épistémologique : des glissements inconscients, chez les élèves, des grandeurs à leurs mesures censées en être le reflet exact entraveraient la mise à distance par rapport aux « faux objets empiriques »-nés de l'illusion que les faits et les observations sont des donnés et non des construits et donc l'accès, au monde 2 des états de conscience dont parle Popper (1973) dans sa modélisation de la rationalité humaine (Schneider, 1988, 2011a). Quant à Job (2011), il montre que cette posture positiviste fait écran à une perception lakatosienne du concept de limite hypothéquant l'entrée des élèves dans l'analyse mathématique dite formalisée. Il s'agit là d'un obstacle récurrent dont Job et Schneider (2014) ont mis en évidence la portée dans de nombreux apprentissages mathématiques relevant de divers niveaux de scolarité et appartenant à des domaines variés.

Mais c'est l'analyse mathématique qui nous intéresse plus particulièrement ici dans la mesure où il s'agit d'un domaine mathématique auquel on initie les élèves dès le lycée mais dont l'enseignement se poursuit dans les universités ou institutions supérieures, qu'il soit adressé à de futurs « matheux » ou ingénieurs ou encore à de futurs utilisateurs potentiels de mathématiques en économie, en chimie, etc. À ce dernier niveau, l'enseignement s'inscrit souvent dans le cadre d'une praxéologie " déduction ", l'axiomatisation des réels y compris au niveau de leur structure topologique autorisant une définition formalisée du concept unificateur de limite sur laquelle se fonde une architecture déductive des 
propriétés préalablement exploitées dans le calcul dit infinitésimal. Les questions sur la pertinence des modèles mathématiques d'objets préconstruits issus de la géométrie ou de la physique n'ont plus droit de cité à ce stade d'étude : il n'y a plus de sens à se demander si le calcul des dérivées donne la valeur exacte d'une vitesse instantanée ou si celui des intégrales permet de calculer avec exactitude une aire curviligne puisque cette vitesse ou cette aire sont désormais définies comme le résultat d'un tel calcul.

Mais ces interrogations sont-elles prises en compte plus tôt, au niveau du lycée par exemple? La réponse à cette question est non, les praxéologies " modélisation » n'étant pas identifiées la plupart du temps, les questions d'élèves telles que celles qui viennent d'être rapportées ne pouvant s'exprimer dans un contrat où les attentes du professeur vis-àvis de ses élèves se résument souvent à des acquisitions procédurales. Les cours contiennent bien, à ce niveau, quelques éléments empruntés à la théorie enseignée à l'université mais ceux-ci sont parcellaires et ne s'intègrent pas dans un discours qui a une cohérence globale. Emblèmes exhibés du travail du mathématicien, ces éléments forment ce que Rouy (2007) a appelé des praxéologies « à trous», soit des organisations mathématiques constituées de tâches (dans les meilleurs des cas) et de techniques mais dont le discours technologique est un semblant de discours :

«Il est en fait constitué du discours théorique proprement dit (tel qu'on peut le voir dans un cours d'analyse universitaire) dans lequel on aurait « fait des trous " pouvant être comblés par le recours aux propriétés visuelles [...] » (p. 252).

Cette chercheuse illustre cette pratique par l'exemple des cours sur les dérivées, habituellement pratiqués au niveau du secondaire, où le lien entre la positivité de la dérivée et la croissance de la fonction n'est jamais démontré bien qu'on énonce quelques théorèmes (celui de Rolle et celui des accroissements finis) qui sont des maillons du cheminement déductif souvent empruntés à l'université pour prouver rigoureusement ce lien. Les maillons emblématiques retenus sont ceux qui se prêtent à des pratiques ostensives éventuellement "déguisées » sous de fausses questions (au sens de Salin, 1999): le professeur montre un objet (ici des graphiques) en misant sur le fait que l'élève y « verra » la même chose que lui. De même, la démonstration du théorème final qui lie positivité de la dérivée et croissance de la fonction sera supprimée au niveau de l'enseignement secondaire et remplacée par le tracé d'une courbe et de tangentes en quelques-uns de ses points. On y juge en effet trop ardue pour les élèves la remontée d'une propriété locale et ayant le statut de condition nécessaire (en un point d'une courbe croissante le signe de la pente d'une tangente est nécessairement positif étant égal à celui d'une sécante parallèle par le théorème des accroissements finis) à une propriété globale ayant le statut de condition suffisante (sans parler d'éventuels points isolés, il suffit que la dérivée d'une fonction sur un intervalle soit positive pour que la fonction y soit croissante). C'est bien cette dernière propriété qui sera utilisée dans l'étude graphique des fonctions mais on ne peut la « montrer " sans tracer des tangentes en chaque point de l'intervalle... les pratiques ostensives ont ici des limites certaines. Certes, le passage mathématique est délicat en ce qu'il suppose la compréhension des propriétés topologiques des nombres réels. Cependant et plus généralement, si l'ostension peut être efficace dans certains cas, elle repose souvent sur un malentendu, les élèves ne « voyant» pas la même chose que le professeur. Par ailleurs, elle n'est pas toujours propre à définir un objet mathématique de manière fonctionnelle. Et c'est bien ce qui se passe pour la dérivée dont l'approche naturalisée au niveau secondaire consiste à l'introduire comme pente de tangente, elle-même " montrée », par ostension, comme "limite de sécantes", aucune topologie ne donnant sens ici au mot « limite». Or, les observations de Cornu (1983), Sierpinska (1985) et Schneider (1988) montrent qu'un tel enseignement se heurte à l'obstacle dit " géométrique » de la limite qui relève, comme le montre Schneider (ibid.), du positivisme empirique. Cet " obstacle empirique » conduit par ailleurs à un cercle vicieux : la dérivée est illustrée comme pente de la tangente, laquelle est rarement définie dans le secondaire comme elle l'est dans la théorie, c'est-à-dire comme droite dont la pente est déterminée par la dérivée...

Cette section illustre donc la difficulté des enseignants du secondaire, qu'ils soient chevronnés ou non, à combler les "déficits » mathématiques inéluctables à ce niveau d'étude autrement que par des gestes d'ostension peu probants. C'est bien un problème de la profession dont on peut supposer qu'il participe au monumentalisme (Chevallard, 
2000) qui affecte aujourd'hui l'enseignement des mathématiques. Mais peut-on agir pour améliorer la situation dès la formation initiale des enseignants ? C'est l'objet de la prochaine section.

\section{Un dispositif de formation dans lequel les élèves-professeurs sont de potentiels vecteurs de changement}

On ne peut, nous semble-t-il, apprécier les tenants et les aboutissants d'une formation initiale sans faire référence aux pratiques observées dans les institutions scolaires qui ont la charge d'enseigner des mathématiques, en particulier les lycées. A-t-on des raisons de penser que l'enseignement des mathématiques s'y déroule au mieux ou, qu'au contraire, il souffre de dysfonctionnements ? Notre sentiment sur les pratiques de terrain rejoint globalement, au-delà des différences individuelles, le constat fait par Chevallard (2000) d'un enseignement des mathématiques s'inscrivant dans une perspective essentiellement « monumentaliste ». On fait « visiter» aux élèves les savoirs mathématiques, comme les pièces d'un musée en occultant souvent les questions auxquelles ces savoirs apportent des réponses. A fortiori ne peut-on mettre en évidence que ces réponses sont situées dans des institutions qui les ont standardisées et que les mêmes questions pourraient être résolues autrement dans d'autres institutions? On peut étendre ce constat sans peine à la Belgique francophone en déplorant, de plus, un repli sans cesse plus marqué sur l'apprentissage des algorithmes, ce repli s'expliquant par la difficulté de trouver un discours de rationalité mathématique adapté au public des élèves du secondaire, sous forme de praxéologies « modélisation »:

« [...] ce premier niveau de rationalité est également non reconnu au niveau des institutions, plusieurs entretiens montrant une sorte de renoncement à toute forme de rationalité dans le secondaire, la répartition : technique au secondaire et théorie à l'université semblant la seule solution » (Rouy, 2009).

Or, s'il est bien un résultat acquis pour les didacticiens des mathématiques, c'est qu'un enseignement systématique des algorithmes, déconnecté d'un travail préalable sur le "sens », est voué à l'échec, ainsi que le souligne Artigue (2007):
« si les recherches didactiques se sont développées, c'est justement parce que cet enseignement-là, tel qu'il était mené, ne donnait pas satisfaction. D’une part, tout en sachant exécuter de façon fiable les dits algorithmes, de nombreux élèves étaient incapables d'en reconnaître les conditions d'emploi dès que cet usage n'était pas induit plus ou moins directement par le contrat didactique, ce qui est le cas dès que l'on sort de la classe de mathématiques, d'autre part, dès que l'usage n'en était pas régulièrement entretenu, les algorithmes étaient oubliés et leur reconstruction impossible à l'élève».

Il semble donc que, en Belgique comme en France à tout le moins, des améliorations assez drastiques soient souhaitables dans l'enseignement des mathématiques.

Mais, est-il crédible que les futurs professeurs soient les vecteurs de telles améliorations? Cela suppose au minimum qu'ils soient conscients des dysfonctionnements de l'enseignement actuel et des pistes possibles d'amélioration. Or, comme le souligne Beckers (2007), c'est une spécificité des futurs enseignants que d'être familiers avec le milieu professionnel dont ils vont faire partie. Bien sûr, ils vont y changer de rôle, passant du statut « d'élève » à celui « d'enseignant », mais les années passées dans l'institution scolaire ont façonné leur représentation a priori de cette institution et même leur posture affective vis-à-vis d'elles. On peut ici convoquer non seulement le concept de « socialisation biographique » de Dubar, cité par Beckers, mais aussi celui « d'assujettissement de sujets à une institution » dont parle Chevallard (1992) dans sa théorie anthropologique. Cet assujettissement se traduit, entre autres, par un sentiment de naturalité des choix faits par les institutions, scolaires en l'occurrence, sentiment qui rend ceux-ci transparents en tant que choix pour les sujets de ces institutions. En particulier, les futurs professeurs ont beaucoup de peine à percevoir comme des choix possibles parmi d'autres les transpositions didactiques standard auxquelles ils ont été habitués en tant qu'élèves, celles-ci étant à ce point naturalisées qu'elles font écran à d'autres possibles. On peut soupçonner là le poids des habitudes potentiellement soumises à des contraintes imaginaires ou non, ou encore le manque d'imagination qui empêche de concevoir d'autres approches.

Ce n'est pas que les futurs professeurs refusent toute forme d'innovation pédagogique. Bien au 
contraire, mus souvent par leur motivation à enseigner, certains semblent croire à des modes d'enseignement différents de ce qu'ils ont connu comme élèves. On peut penser qu'il y a là quelque espoir de pouvoir miser sur eux pour commencer à faire vivre certaines innovations dans les institutions scolaires ou, à tout le moins, en faire comprendre les enjeux. Cependant, le regard des futurs professeurs est, d'emblée, relativement naif ce qui laisse craindre une adhésion bien superficielle à des dispositifs dont ils ne maîtrisent ni la portée potentielle, ni les limites, se retranchant souvent au niveau des valeurs identifiées par Margolinas (2004) pour évoquer, de manière fort allusive et très peu argumentée, que telle approche est plus « concrète » qu'une autre ou qu'elle va « motiver » les élèves parce qu'elle montre que les dérivées servent dans d'autres disciplines. On rejoint là des observations faites et analysées par Schneider (2006a, 2006b, 2011b) pour montrer que les réformes d'enseignement sont introduites par la noosphère sur un mode essentiellement idéologique et reçues par les acteurs de terrain sous forme d'une adhésion ou d'un rejet "épidermiques ». C'est le cas, en particulier, de la réforme axée autour de la notion de compétence légitimée par des références «socio-constructivistes» qui sont, comme l'analyse Schneider (ibid.), des conversions indues en injonctions institutionnelles d'enseignement de théories comme celles de Piaget. Or, ces théories se situent dans un autre cadre, en l'occurrence celui de la psychologie génétique où il est plus question de modéliser le développement de l'intelligence chez l'enfant et l'adolescent que d'étudier les apprentissages scolaires induits par tel ou tel type d'enseignement. Il en résulte des slogans non questionnés tels que « l'élève doit être acteur de son propre apprentissage » ou « il faut remplacer le cours frontal par des activités ». Et, lors de notre formation et des évaluations ou travaux associés, nous observons tous les futurs enseignants en formation initiale relayer de tels slogans, certains évoquant tout au plus une réserve quant au fait que « commencer par des situations-problèmes prend trop de temps par rapport au programme à voir ». S'agit-il là d'un effet de contrat ? Ces étudiants sont-ils imprégnés de cette idéologie lors de leurs stages sur le terrain, de par leur propre expérience d'élève dans le secondaire ou par le biais d'autres enseignements en sciences de l'éducation lors de leur formation initiale? Nous ne le savons pas et il ne nous appartient pas d'en juger.
De toute façon, ce n'est pas cette investigation qui nous intéresse ici.

C'est sur base de l'ensemble de ces constats négatifs et dans l'idée - l'illusion sans doute, nous y reviendrons - de faire des élèves-professeurs de potentiels "vecteurs de changement " que nous avons conçu, au fil du temps, une formation en didactique des mathématiques qui prend place dans un contexte institutionnel décrit plus loin. Voici les principes organisateurs de cette formation qui vise globalement deux objectifs : favoriser chez les formés une posture plus intellectualisée et ouvrir leur conscience à un champ plus vaste de possibles.

D'une part, cette formation se fonde sur des théories didactiques que nous enseignons dans une perspective qui se veut non prescriptive. En particulier, la théorie des situations didactiques (TSD) de Brousseau (1998) y est présentée comme un réseau conceptuel susceptible d'analyser tout dispositif d'enseignement quelle qu'en soit la forme, que celui-ci soit ou non inspiré du paradigme socio-constructiviste. En outre, nous insistons sur le fait que cette théorie n'a pas pour but de montrer les avantages d'un enseignement inspiré de ce dernier paradigme mais qu'elle permet d'en formuler des conditions sine qua non de son fonctionnement en situation scolaire. Parmi ces conditions qui caractérisent les situations adidactiques, citons brièvement le caractère fondamental des problèmes soumis aux élèves par rapport au savoir à construire, l'existence d'un milieu et d'un contrat qui en permettent la dévolution sans jouer trop sur des « effets » de contrat, la part irréductible d'institutionnalisation faite par le professeur et le caractère collectif de la construction du savoir. Ensuite, cette même théorie permet aussi d'évaluer les opportunités d'un enseignement socio-constructiviste, en termes d'identification et de franchissement d'obstacles d'apprentissage. Le choix éventuel d'un dispositif d'enseignement socio-constructiviste et sa gestion supposent alors des analyses, au cas par cas, dont les dimensions épistémologique et didactique sont conséquentes. Quant à la théorie anthropologique du didactique (TAD) de Chevallard (1992), elle permet de questionner la relativité institutionnelle du savoir et le processus de transposition à l'œuvre dans les praxéologies mathématiques en vigueur sur le terrain, praxéologies que l'on se doit de dénaturaliser pour en mieux cerner les enjeux et les limites. Cette modalité de formation relève, nous semble-t-il, de ce que Houdement et Kuzniak (1996) 
nomment, parmi plusieurs stratégies de formation, une "stratégie basée sur la transposition». Elle se caractérise en effet par une " professionnalisation basée sur la didactique » (ibid.) et les savoirs visés sont des « savoirs en voie de constitution [ou constitués ajouterions-nous] attachés à des écoles de didactique $»$ (ibid.).

D'autre part, sur un plan plus pratique, cette formation croise deux approches. La première est une approche bottom up, ainsi appelée par Cirade (2006), pour désigner la place octroyée dans la formation professionnelle à l'étude de questions « ombilicales », c'est-à-dire proposées par les formés comme questions émanant de leur vécu quotidien. Dans cette perspective, nous nous appuyons, lors de nombreuses séances, sur des "pièces rapportées » par les élèves-professeurs de leur pratique, soit des faits divers rencontrés sur le terrain, que ce soit à l'occasion de cours donnés par un professeur en fonction et qu'ils ont eu l'occasion d'observer ou lors de cours qu'ils ont donnés eux-mêmes lors de stages. Ce peut être des observations sur des erreurs ou réactions d'élèves qui les étonnent ou sur des choix didactiques en vigueur dans les écoles où ils effectuent leurs stages et qu'ils questionnent. Il s'agit alors, au cours de didactique, d'élaborer collectivement des réponses supposées viables, éventuellement en rupture par rapport aux réponses standardisées dans les pratiques enseignantes mais que l'on aura accepté de déconstruire et de reconstruire. L'autre approche est de confronter les futurs enseignants avec des ingénieries didactiques qui possèdent plusieurs caractéristiques. Elles sont issues de la recherche, ayant été conçues comme méthodologies propres à engendrer des observables, d'une part, sur de possibles milieux susceptibles de favoriser chez les élèves la production de modèles mathématiques sous des formes parfois embryonnaires et, d'autre part, sur les obstacles d'apprentissage relatifs à cette construction. Ces ingénieries s'inscrivent dans une transposition qui se différencie sensiblement des transpositions habituelles sur un thème mathématique donné et, pour celles qui nous intéressent dans cet article, relèvent d'une praxéologie " modélisation ». L'articulation de ces deux approches donne prise à une dialectique " médias / milieux » qui est, pour Chevallard (2007), une " condition cruciale pour qu'un processus d'étude et de recherche ne se réduise pas au recopiage acritique d'éléments de réponse épars dans les institutions de la société ».
Les ingénieries et leurs effets sur les apprentissages constituent ici des medias « adressés » par le monde de la recherche au public des futurs professeurs et susceptibles de faire un milieu de référents pour leur dévoluer la construction de leurs propres réponses. Dans une telle optique, les ingénieries en question constituent à la fois un matériau sur lequel les étudiants apprennent à faire des analyses didactiques et un tiers-objet censé provoquer un phénomène «d'estrangement $»^{2}$ (Ginzburg, 2001) pour leur faire prendre conscience d'un espace de liberté qu'ils ne soupçonnent pas forcément a priori. Cependant, un écueil a priori à la réalisation de cette entreprise est lié au contrat didactique : c'est nous qui proposons les ingénieries en question (voire les concevons mais, de cela, les étudiants ne sont pas forcément au courant) et, bien sûr, le risque de leur réception par les formés sur un mode normatif est loin d'être négligeable. C'est là que jouent encore les théories didactiques comme réseaux conceptuels, lesquelles théories outillent les étudiants dans le but de les aider à problématiser toute question d'apprentissage ou d'enseignement et, en particulier, d'analyser a priori les variables didactiques d'une situation donnée et de leurs possibles impacts sur les réactions des élèves.

Enfin, nous misons sur les théories didactiques aussi pour faire des formés des partenaires de la réflexion didactique, au-delà d'une posture prescriptive. En effet, elles nous permettent d'afficher nos propres cadres de pensée, ainsi que les non-négociables de la formation qui relèvent surtout d'une posture réflexive et d'une rigueur d'analyse.

\section{MÉTHODOLOGIE D'OBSERVATION, OBSERVABLES ET ANALYSE}

Les principes de notre formation et le choix de valeurs sous-jacentes ayant été mis à plat, nous sommes à présent en mesure d'y situer une expérimentation, ainsi que d'analyser les observables récoltés à la lumière du cadre théorique développé plus haut.

Auparavant, nous décrivons le public des élèvesprofesseurs concernés et la structure globale de leur formation professionnelle. 
Maggy Schneider E Pierre Job

\section{Le public des élèves-professeurs}

En Belgique, l'université ne forme que les futurs enseignants de lycée (trois dernières années de l'enseignement secondaire). La formation professionnelle prévue a un poids de 30 ECTS (norme européenne) qui couvre une demi-année académique et qui, dans la Faculté des Sciences concernée ici, est étalée sur un an (ou deux si l'étudiant décide de fractionner). Il y a deux publics d'étudiants. Ceux qui préparent le master en mathématique à finalité didactique : ils ont eu une formation en mathématique pendant 4 ans et, lors de la $5^{\mathrm{e}}$ année, poursuivent cette formation en même temps qu'ils assument la formation professionnelle. Ils doivent aussi faire un mémoire de fin d'études qui peut être un mémoire professionnel mais pas forcément. L'autre public est constitué d'ingénieurs civils ou industriels déjà diplômés dont certains ont travaillé (ou travaillent encore) dans le secteur privé. Ils font la formation pour enseigner et obtiennent en un an ou deux un diplôme qui complète leur titre d'ingénieur. Il n'y a aucun concours en Belgique, l'évaluation de la formation professionnelle relève de l'université qui dispense la formation.

Cette formation professionnelle comporte plusieurs volets entre lesquels se répartissent les 30 crédits selon la pondération suivante dans notre université :

- Analyse de l'institution scolaire et politiques éducatives : 1 crédit ;

- Éducations aux médias : 1 crédit ;

- Éléments de sociologie de l'éducation : 1 crédit ;

- Diversité des publics scolaires : 3 crédits;

- Éthique professionnelle et formation à la neutralité et à la citoyenneté : 2 crédits ;

- Psychologie éducationnelle de l'adolescent et du jeune adulte : 2 crédits;

- Séminaire d'approche interdisciplinaire :

1 crédit ;

- Pédagogie générale, y compris des stages d'observation et pratiques réflexives : 4 crédits ;

- Didactique des mathématiques : 7 crédits ;

- Stages (stages d'observation et stages d'enseignement effectif sous la guidance du maître d'un maîtres de stages) : 8 crédits.

Nous gérons donc la moitié des crédits (didactique et stages) en tâchant d'articuler cours de didactique et stages, comme nous l'avons décrit supra. Ajoutons ici qu'un travail écrit et individuel de retour réflexif sur les stages réalisés est demandé aux formés, avec des demandes précises de notre part basées sur nos propres observations des cours qu'ils ont prestés sous des contraintes souvent très fortes imposées par les maîtres de stages. Nous testons alors leur réflexivité a posteriori vis-à-vis des choix didactiques qu'ils ont faits ou qui leur ont été imposés et même vis-à-vis de la transposition induite par les programmes scolaires et les documents officiels qui les accompagnent. C'est à cela que nous ferons allusion plus loin en parlant de réflexivité à propos des stages.

C'est dans ce contexte que nous faisons étudier à nos élèves-professeurs des ingénieries construites à des fins de recherche, ainsi que les observables que celles-ci ont permis de mettre à jour. Ces observables sont relatifs tantôt aux obstacles d'apprentissage - tels ceux décrits plus haut - tantôt aux formes embryonnaires des savoirs telles qu'elles peuvent être construites par les élèves dans une praxéologie " modélisation», par exemple un calcul de dérivée qui consiste à annuler un incrément de la variable dans une expression algébrique simplifiée. Et c'est dans le même contexte que nous avons recueilli les données relatives à la présente étude sur la manière dont des élèves-professeurs appréhendent des ingénieries issues de la recherche. Comme dit dans l'introduction, ces ingénieries servent ici de méthodologies de recherche supposées créer, dans le contexte de la formation de futurs enseignants, des observables qui deviendront phénomènes à la lumière des cadres théoriques retenus. Cependant, ces mêmes ingénieries ont déjà joué le rôle de méthodologies de recherche ayant précédemment permis de mettre en évidence des phénomènes didactiques au niveau des élèves cette fois. Et il se fait que les observables récoltés ici ne peuvent être interprétés qu'à la lumière de ces derniers phénomènes. Ce qui nous oblige à articuler des niveaux d'analyse : le premier relatif au travail des élèves de lycée, l'autre concernant les réactions des élèves-professeurs dans le cadre de la formation concernée. Ces analyses successives font l'objet des sections qui suivent.

Un premier travail d'ordre méthodologique : le choix d'un thème mathématique et l'analyse a priori du matériau soumis aux formés

Le thème choisi relève de l'analyse mathématique, domaine dont nous avons justifié plus haut l'intérêt 
pour la présente étude. Rappelons qu'il existe un partage assez net entre la transposition en vigueur dans l'enseignement secondaire qui s'apparente à ce qu'on a appelé le calcul infinitésimal dans l'histoire et les cours universitaires relevant de l'analyse dite formalisée dont le système de validation repose sur une définition du concept de limite en termes d'inégalités et de quantificateurs. Il permet donc $a$ priori d'observer dans quelle mesure et comment les étudiants parviennent à éviter les praxéologies « à trous » mais aussi des cercles vicieux liés à une vision empiriste. En amont, il doit permettre d'étudier si les formés arrivent à prévoir sous quelle(s) forme(s) embryonnaires, dans une praxéologie "modélisation » préalable à une standardisation théorique, les élèves sont susceptibles de construire le savoir visé. Il s'agit aussi de voir si les élèves-professeurs sont capables d'identifier les variables didactiques des problèmes ayant provoqué ces apprentissages. C'est ce qui a déterminé le choix du domaine mathématique. Le choix du thème, quant à lui, est dicté par une analyse épistémologique qui en montre la portée, ainsi que le souci de faire travailler un couple " question / réponse » : ici, c'est une question relative à un débit ou une vitesse variable qui va motiver la construction de la dérivée laquelle, comme chez Newton, émergera d'un contexte cinématique pour servir ensuite de marchepied au taux de variation instantané général dans lequel la variable indépendante n'est pas forcément le temps.

Deux approches distinctes ont été proposées aux élèves-professeurs selon des modalités que nous préciserons dans la prochaine section. Ces approches sont construites, l'une et l'autre, autour d'un problème-clé qui s'inscrit dans un parcours d'étude plus global. C'est sur ces problèmes que nous nous focaliserons en l'espace de cet article.

Voici l'énoncé du premier problème : «Une pompe alimente un vase conique (FIG. 1). Elle est réglée de telle manière que le niveau de l'eau y monte régulièrement de $1 \mathrm{~cm} / \mathrm{min}$. L'angle au sommet du cône vaut $90^{\circ}$. Jusqu'à quand le débit de la pompe sera-t-il inférieur à $100 \mathrm{~cm}^{3} / \mathrm{min}$ ?»

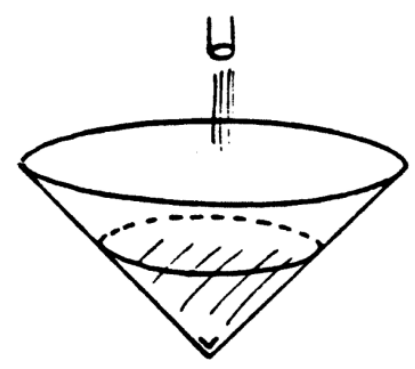

FIG. 1 - Vase conique.

Et celui du second : " On imagine deux particules ponctuelles, P1 et P2 (FIG. 2) qui se meuvent sur un axe orienté muni d'une origine et d'une unité. Voici les graphiques qui représentent, à tout moment, leur position sur la trajectoire. À quel moment les deux mobiles ont-ils la même vitesse? " Dans un premier temps, seuls les graphiques sont donnés. Ensuite, on précise des expressions algébriques: d'abord une loi du second degré pour le mouvement non uniforme, puis, dans un second temps, on la remplace par une loi du troisième degré.

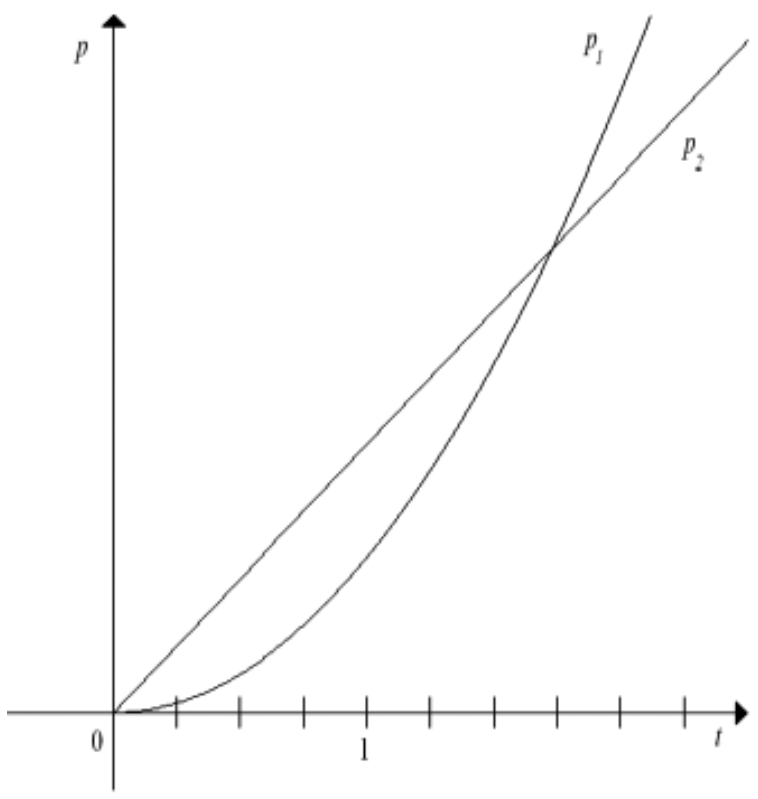

FIG. 2. - Lois de mouvements.

Ces deux problèmes possèdent des caractéristiques communes :

- deux vitesses sont mobilisées, l'une constante, l'autre non. En outre, c'est la constance de l'une qui induit l'intuition de la variabilité de l'autre. Dans le problème du vase conique, la hauteur $h$ de l'eau évolue à vitesse constante et, le vase s'élargissant, le 
débit ne peut qu'augmenter constamment. Dans le second problème, c'est le contraste entre le caractère rectiligne d'un graphique et le caractère curviligne de l'autre qui joue et nous y reviendrons ;

- dans les deux cas, l'inconnue est, non pas une vitesse, mais un temps dont l'existence est acceptée d'entrée de jeu par les élèves sur base d'une hypothèse implicite de continuité, soit cinématique, soit graphique ;

- il en découle, chaque fois, un engagement d'un calcul de taux moyen, une mise en cause de ses limites, l'idée de travailler sur des intervalles de temps de plus en plus petits et, in fine, l'identification d'un nouveau calcul qui consiste à annuler l'intervalle de temps dans l'expression du taux moyen, une fois faites toutes les simplifications algébriques. Ce nouveau calcul, celui d'une dérivée non théorisée encore, est susceptible de faire l'objet d'un débat entre les élèves pour des raisons telles que « en un temps nul, aucun volume n'est versé » ou « on ne peut mesurer une vitesse instantanée, car rien que chronométrer prend du temps »;

- enfin, les deux contextes se prêtent à une validation pragmatique de ce nouveau calcul en ce qu'il permet de trouver la réponse exacte à la question posée : grâce à une expérience mentale mobilisant un autre vase cylindrique dans le premier cas et, dans le second, grâce au fait que le résultat obtenu peut être corroboré par la mise en ouvre d'autres stratégies déjà standardisées dans l'exemple où le mouvement non uniforme est uniformément accéléré.

Mais, au-delà de ces points communs, les deux problèmes se différencient à plus d'un égard, les contextes et caractéristiques de « surface " n'étant pas les mêmes :

- dans l'un, c'est un débit qui varie et, dans l'autre, c'est la vitesse d'un mobile ;

- à propos du vase conique, le registre graphique n'est pas d'office mobilisé ; il l'est, par contre, dans le problème des particules et on peut supposer que ce dernier problème se prête mieux et même spontanément à l'étude des tangentes;

- dans le premier problème, le mouvement est décrit d'une manière «brute » par le biais d'une expérience de pensée ; dans le second, les mouvements sont précisés de manière plus symbolisée par le biais de graphiques puis d'expressions analytiques.
Cette dernière différence est de taille et explique que ce deuxième problème doit être précédé d'un autre qui permet aux élèves de travailler des intuitions propres à leur faire comprendre que le caractère curviligne d'une loi de mouvement traduit la variabilité de la vitesse. Il s'agit de demander aux élèves de décrire le plus complètement possible le mouvement d'une particule dont la loi de mouvement, exprimant la position par rapport au temps, est donnée par la figure 3 :

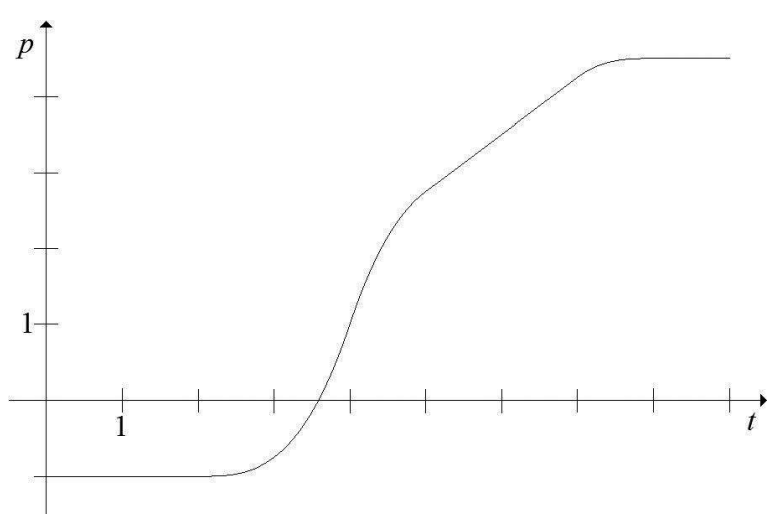

FIG. 3. - Mouvement d'une particule.

Outre la confusion entre la loi de mouvement et la trajectoire du mobile, un tel graphique permet de faire travailler le caractère intensif de la vitesse : après une période où le mobile est à l'arrêt et a, par conséquent, une vitesse nulle, on peut dire que sa vitesse augmente d'abord pour devenir constante ensuite, puis enfin diminuer jusqu'à s'annuler. Ce problème favorise ainsi l'intuition que la variation de la vitesse est liée à la "pente de la courbe », comme disent les élèves.

Ces problèmes ont été testés dans des classes et des recherches ont permis de valider l'impact des variables didactiques choisies en termes d'identification et de questionnement, par les élèves, d'un nouveau calcul qui préfigure celui des dérivées (Schneider, 1992 ; Gantois \& Schneider, 2012). Depuis une quinzaine d'années, ils font partie intégrante d'un dispositif d'initiation à la didactique en même temps qu'ils permettent de mettre en évidence des observables liés aux difficultés sus-mentionnées des futurs professeurs. Rappelons que cette initiation à la didactique vise, entre autres, à instruire le regard que les futurs enseignants peuvent avoir sur l'idéologie des situations-problèmes d'éléments théoriques qui en font voir les potentialités mais aussi les limites, 
en particulier le caractère fondamental et le caractère adidactique de ces situations. Nous les illustrons ci-dessous à propos des problèmes précédents pour permettre au lecteur de situer les réactions d'étudiants par rapport à l'analyse a priori. Le problème du vase conique possède un caractère fondamental par rapport au concept de débit instantané car il ne peut être résolu sans ce dernier (qu'il soit ou non en construction). Celui des particules, par contre, ne possède pas ce caractère vis-à-vis du concept de vitesse instantanée dans le cas où le mouvement non uniforme est donné par une fonction du $2^{\mathrm{e}}$ degré. En effet, dans ce cas, les élèves concernés peuvent répondre à la question en l'évitant, soit en calculant, par une équation du second degré, l'instant auquel une droite dont la pente est la vitesse constante du mouvement uniforme rencontre en un seul point l'autre loi de mouvement, soit en déterminant l'instant en lequel l'écart entre les mobiles est maximal ce qu'ils sont capables de faire, l'écart étant donné dans ce cas par une fonction du second degré. Par contre, les connaissances des élèves à ce stade ne leur permettent pas de pareilles stratégies dans le cas où le mouvement non uniforme est donné par une fonction du $3^{\mathrm{e}}$ degré et la question a alors un caractère fondamental supposant la construction de la vitesse instantanée. Mais, pour être dévolue aux élèves, une situation-problème doit avoir un caractère adidactique, ce qui suppose en plus l'existence d'un milieu dans lequel les élèves trouvent des référents pour évaluer leurs propres stratégies. De ce point de vue, les caractéristiques du problème du vase conique (la constance de la vitesse de la montée de l'eau, l'élargissement du vase...) jouent un rôle aux côtés des connaissances antérieures des élèves et des débats entre eux que le professeur autorisera. Le problème relatif à la description d'un mouvement (FIG. 3) installe, lui, des intuitions graphiques sur les mouvements, par exemple le fait de lier accélération et concavité tournée vers le haut, qui « feront milieu » pour les élèves au moment où ils aborderont le problème des deux particules. Quant à ce dernier, le cas où le mouvement non uniforme correspond à une fonction du second degré fournit un milieu de validation pragmatique pour l'autre, au sens décrit supra, étayant ainsi la validité d'un calcul sujet à caution.

\section{Les modalités du travail proposé aux élèves- professeurs et du recueil de données}

Le premier problème, analysé dans la section précédente, celui du vase conique, a donné lieu à un travail en présentiel. À ce moment, les élèvesprofesseurs avaient déjà reçu un enseignement sur la théorie des situations de Brousseau : situation fondamentale, didactique, dévolution, milieu, contrat, variable didactique et institutionnalisation. Par petits groupes de deux, ils ont été invités à résoudre le problème du vase conique puis à faire une analyse a priori de ce problème, le public des élèves potentiels leur ayant été précisé. Une institutionnalisation (annexe 1) a fait suite à leur recherche: on y a relaté des réactions avérées d'élèves et on les a analysées en termes de milieu, en lien avec les variables didactiques du problème, comme illustré ci-dessus. Par ailleurs, un enseignement sur les obstacles d'apprentissage a été dispensé à ce moment, entre autres sur l'obstacle géométrique de la limite et le cercle vicieux auquel il conduit, dans les manuels, à propos de la tangente (voir supra). Le problème du vase conique, ainsi qu'un travail des étudiants sur la géométrie vectorielle et un autre sur le concept de limite ont servi également d'introduction aux praxéologies «modélisation » et « déduction ».

La seconde approche de la dérivée, analysée dans la section 2.1, a fait l'objet d'une évaluation « à blanc » (qui ne relève pas de l'évaluation certificative) devant aider les étudiants à se situer par rapport à l'objectif annoncé d'analyse didactique. Cette deuxième approche a été communiquée trois semaines auparavant, assortie de questions destinées à alimenter leur réflexion. Les élèves-professeurs ont eu alors à lire un projet d'enseignement commençant par les problèmes relatifs aux figures 2 et 3 et les institutionnalisations de savoir correspondantes pour les élèves (annexe 2), mais contenant aussi d'autres aspects et applications de la dérivée : problèmes d'optimisation et exploitation de la tangente dans le cadre d'une approximation affine locale d'une fonction, ce dernier aspect étant plutôt absent des manuels et des pratiques enseignantes. On leur a annoncé un test à blanc sur ce projet et sur l'analyse didactique qu'on peut en faire, leur proposant les questions suivantes pour étayer leur lecture :

- Vous imaginez, qu'à propos de la figure 2, on dévolue aux élèves la question suivante: « À quel moment les mobiles $\mathrm{P}_{1}$ et $\mathrm{P}_{2}$ ont- 
ils la même vitesse ?». On précise, dans un premier temps, les lois de position par des graphiques et, dans un second temps, par des expressions analytiques. La loi $\mathrm{p}_{1}(\mathrm{t})$ est d'abord la fonction $\mathrm{t}^{2}$, ensuite la fonction $\mathrm{t}^{3}$. Cette situation, dans sa globalité, possède-telle un caractère fondamental par rapport au savoir visé ? Développez.

- Pourquoi définir $\mathrm{p}_{1}(\mathrm{t})$ d'abord par une fonction du second degré, ensuite par une fonction du troisième degré ?

- Quelles sont les variables didactiques de cette situation qui créent un milieu permettant de la rendre partiellement adidactique? Faites un parallèle avec la situation du vase conique : ce qui les rapproche, ce qui les distingue.

- Le deuxième extrait de l'annexe concerne une situation (FIG. 3) qui se situe en amont de celle que vous venez d'analyser. On y demande aux élèves de décrire, avec le maximum d'informations, le mouvement du mobile correspondant au graphique donné. Discutez du rôle et la pertinence de cette situation.

Le test à blanc, quant à lui, a porté sur les questions suivantes :

- Supposez, qu'à propos de la figure 2, on demande aux élèves : "À quel moment les mobiles $\mathrm{P}_{1}$ et $\mathrm{P}_{2}$ ont-ils la même vitesse si la loi de position de $\mathrm{P}_{1}$ est donnée, d'abord par la fonction $\mathrm{t}^{2}$, ensuite par la fonction $\mathrm{t}^{3}$ ? ». Dans cette question, on compare deux mouvements dont l'un est uniforme et l'autre non. Pourquoi ? Dans un premier temps, le mouvement non uniforme est déterminé par une fonction du second degré et, plus tard, à la page 15, par une fonction du troisième degré. Quel est le rôle joué par chacun de ces deux cas ? Expliquez.

- Cette situation, dont mention est faite à la question 1 , possède-t-elle un caractère fondamental par rapport au savoir visé ? Développez.

- Quelles sont donc, en bref, les variables didactiques de cette situation qui émergent de vos réponses aux questions 1 et 2 et quels en sont les équivalents dans le problème du vase conique?

- Toujours par rapport à cette situation, quel rôle joue la première activité proposée aux élèves et l'institutionnalisation qui l'accompagne ? Cette activité consiste à décrire, le plus complètement possible, le mouvement donné par le graphique de la figure 3.
- Comment l'enseignement de la tangente estil conçu dans l'ensemble du projet ? Se différencie-t-il de la transposition habituelle telle qu'apparente dans la plupart des manuels ? En quoi ?

\section{Les observables récoltés et leur analyse}

Les étudiants ont réalisé individuellement ce test à blanc qui a duré deux bonnes heures et ont été invités à expliquer leurs arguments par écrit. Voici un échantillon constitué d'exemples de leurs réponses qu'il s'agit d'apprécier en fonction de l'analyse didactique des problèmes faite supra.

Commençons par des propos d'étudiants qui ne sont pas vraiment rentrés dans le jeu de l'analyse didactique :

- «Aussi bien ce problème que celui du vase conique ont pour but d'arriver au concept de dérivée. Dans les deux cas on parle de vitesse instantanée et de dérivée ";

- « Passage de la connaissance de vitesse moyenne à une première approche de la connaissance d'une vitesse variable instantanée ;

- « Avantage de relier un concept physique (vitesse ?) avec sa représentation graphique et son expression algébrique »;

- « Le choix des fonctions $\mathrm{t}^{2}$ puis $\mathrm{t}^{3}$ établit une gradation dans les difficultés de calculs algébriques pour le calcul de $\Delta \mathrm{p}_{1}$ et $\Delta \mathrm{p}_{2} "$; - « Le choix des fonctions $\mathrm{t}^{2}$ et $\mathrm{t}^{3}$ permet d'établir les premières formules de dérivation »; - « Lélève s'approprie la notion de vitesse, les tâches sont déclinées en activités qui ont du sens et qui peuvent motiver l'élève, une solution est possible»;

- «La question posée ici est trop difficile pour que les élèves puissent y répondre sans l'intervention du professeur. Moyennant des sous-questions orientées, le problème posé peut être considéré comme situation fondamentale».

On n'y voit pas trace d'une description des variables didactiques des problèmes et de leur possible impact sur les stratégies des élèves, encore moins une analyse. Seul leur enjeu global est exprimé : la dérivée et, conformément aux programmes scolaires, son interprétation cinématique en termes de vitesse instantanée mais cet enjeu-là était annoncé dès le début du travail. D'autres arguments font référence 
aux aspects procéduraux, comme le calcul même des fonctions dérivées. D'autres encore, plus habituels, semblent relever du niveau +3 de Margolinas (2004) relatif aux valeurs et conceptions sur l'enseignement / apprentissage : les changements de registre favorisent l'apprentissage, les activités sont porteuses de sens et de motivation pour l'élève, les calculs se doivent d'être proposés par ordre de difficulté croissante, la technique des sous-questions rend la tâche plus accessible. Mais ces propos ne sont pas argumentés au sein du contexte spécifique.

Nous reviendrons sur la proportion des étudiants qui ne rentrent pas vraiment dans l'analyse des variables didactiques pour en venir directement aux autres et illustrer, toutes questions confondues, en quoi ils parviennent à transposer à une approche ce qui a été analysé pour l'autre, en transférant ainsi des concepts didactiques plus généraux. En même temps, nous montrons que c'est l'analyse a priori qui donne les indicateurs d'une réelle entrée dans l'analyse des problèmes.

Commençons par le caractère fondamental des questions soumises à l'analyse. Par rapport au savoir visé, ce caractère ne peut être jaugé qu'en considérant toutes les fonctions mobilisées, la présence d'une fonction du $3^{\mathrm{e}}$ degré invalidant les stratégies autres que la dérivation. C'est bien ce que certains expriment :

- « La question relative au second degré n'a pas de caractère fondamental puisqu'une solution existe sans avoir recours à la dérivée. Pour le troisième degré, la dérivée devient utile et répond à un besoin de généralisation $»$;

- « Caractère fondamental par rapport au savoir visé dans le cas de $\mathrm{t}^{3}$ car seule la méthode qui aboutit à la dérivée donne la réponse à la question posée. Le nouveau savoir constitue une économie de pensée et permet de résoudre un (de) nouveau(x) problème(s). Il devient donc incontournable »;

- « Le choix de la fonction $\mathrm{t}^{3}$ permet de généraliser la méthode en $\Delta t$ et à la faire accepter, à en percevoir l'intérêt et à institutionnaliser le savoir. En effet, les autres méthodes ne sont pas assez performantes puisqu'aucune validation n'est possible dans ce cas, les techniques de résolution d'équations du troisième degré et d'optimisation de fonctions du troisième degré n'étant pas disponibles. On ne peut donc pas dans ce cas trouver l'équation de la tangente $»$.
Plusieurs stagiaires sont conscients de la forme embryonnaire que prend le calcul des dérivées à ce stade et des réserves qu'elle est susceptible de susciter chez les élèves, la suppression de termes contenant $\Delta \mathrm{t}$ ne s'accompagnant d'aucune compensation algébrique standard :

« Les deux problèmes favorisent l'interaction entre l'idée d'un nouveau calcul en opposition avec les règles de résolution des équations (lorsqu'on annule $\Delta \mathrm{t}$ ) et l'intuition physique. Lidée de poser $\Delta \mathrm{t}=0$ pose un problème en mathématique mais pas forcément en physique. »

Mais aussi que ce nouveau calcul sujet à caution peut faire l'objet d'une validation pragmatique à propos de la loi de mouvement du second degré dont la présence se justifie ainsi :

- « On peut valider le résultat obtenu et donc, si le fait d'annuler $\Delta \mathrm{t}$ peut paraître scandaleux, on a d'autres moyens pour vérifier le résultat $»$;

- «Dans la méthode en $\Delta \mathrm{t}$, on simplifie d'abord par $\Delta \mathrm{t}$ (non nul) puis on le supprime dans un membre sans le compenser dans l'autre membre (on le considère donc nul) : est-ce que ça passe auprès des élèves ? Le choix de la fonction $\mathrm{t}^{2}$ permet de vérifier le résultat obtenu en $\Delta \mathrm{t}$ par d'autres moyens : optimiser une fonction du second degré ou imposer qu'une fonction du second degré possède une racine double. Cette fonction est un cas simple de dérivée variable »;

- « Le mouvement uniforme (droite sur le graphe) permet la justification par les droites de pente 3 et rend simple la validation par maximalisation des écarts de position (pour la fonction $\mathrm{t}^{2}$ )».

Ce sont là des caractéristiques propres aux praxéologies « modélisation » dont on sait qu'elles sont étrangères à la plupart des pratiques enseignantes (Rouy, 2007). La présence de telles caractéristiques dans les propos de certains formés constitue donc un indice de leur compréhension de certains enjeux du projet.

Les variables didactiques sont, chez ces élèvesprofesseurs, bien identifiées et correctement mises en correspondance avec celles du problème du vase conique. D'abord, le contraste possible entre la constance d'une vitesse et la variabilité de l'autre :

- « Dans les deux problèmes, des vitesses sont liées. Une vitesse constante et une vi- 
tesse variable sont des variables didactiques de la situation. On peut mettre en parallèle la variable "vitesse constante " avec la donnée numérique « la vitesse de montée de l'eau vaut $1 »$;

- « On compare un mouvement uniforme avec un mouvement non uniforme; dans l'exemple du vase conique, on fait la même chose pour un débit. Le fait qu'une des grandeurs varie de façon constante permet de mettre en évidence que l'autre n'évolue pas à vitesse constante »; - « On propose chaque fois un problème où les élèves doivent mettre à l'épreuve l'idée de vitesse constante; la vitesse variable mène à la vitesse instantanée ».

Ensuite, l'existence d'un milieu qui permet d'interpréter l'accélération d'un mobile en termes de concavité d'un graphique et de favoriser la mise à l'épreuve du concept de vitesse moyenne par la considération d'intervalles de temps de plus en plus petits :

- « Le lien entre la concavité et les vitesses qui augmentent ou diminuent fait ressortir le fait que la vitesse moyenne ne suffit pas et l'importance de prendre des intervalles de temps de plus en plus petits »;

- « Découpage du temps en intervalles de plus en plus fins qui conduit les élèves à considérer des taux de variation moyens ».

Enfin, le fait que l'inconnue du problème est le temps et non pas la vitesse :

- « Dans les deux situations, c'est le concept de vitesse instantanée qui est présenté mais au lieu de demander aux élèves la vitesse ou le débit à un moment donné, on leur demande le moment auquel la vitesse ou le débit sera égal à une quantité donnée, ce qui leur paraît plus réaliste. Les élèves ne doutent pas du fait qu'il y a une solution pour chacune des questions : il existe bien un moment où le débit sera de $100 \mathrm{~cm}^{3} / \mathrm{min}$; pour le mobile, vu qu'ils ont résolu le problème pour $\mathrm{t}^{2}$ ils se doutent qu'une solution existe aussi pour $\mathrm{t}^{3} »$.

Quant à l'étude de la transposition didactique en vigueur (question 5), plusieurs élèves-professeurs arrivent à contraster, sur la base d'indices précis, le sort fait au concept de tangente dans le texte qu'ils doivent analyser et les dysfonctionnements observés dans les manuels, en termes de cercle vicieux lié à une conception empiriste de la tangente ou d'occultation de certains aspects :
- « La définition de la tangente fait appel à la dérivée alors que l'inverse est présenté dans les manuels »;

- « Dans les manuels la tangente est souvent présentée comme une droite ayant un seul point d'intersection avec la courbe. Si c'est vrai pour le cercle, cette définition n'est plus valide pour les coniques par exemple»;

- "Manuels : enseignement de la tangente conçu « limite » de sécantes; confusion avec " limite géométrique » de sécantes et limite numérique symbolisée du quotient différentiel ; la pente de la tangente passe au second plan. Cette approche constitue un obstacle épistémologique qui est évité dans le projet. En effet, ici on définit d'abord la dérivée en se basant sur son utilité à résoudre un problème et ensuite la tangente en un point d'une courbe comme la droite qui passe par ce point et dont le coefficient angulaire vaut la dérivée de la fonction en ce point. On part de la dérivée qu'on associe à une pente pour en arriver au concept de tangente $»$;

- «La tangente peut être utilisée comme droite qui aide à connaître la forme globale d'une courbe (droite guide) et pour faire des approximations (droite outil d'estimation). Dans les manuels, ces utilisations sont rejetées bien loin dans le cursus et sont vues comme applications des dérivées et non comme des outils».

Ces quelques résultats permettent de distinguer et de caractériser deux populations d'élèves-professeurs : ceux qui en restent à des arguments convenus empruntés aux idéologies d'enseignement ambiantes et ceux qui rentrent dans une analyse didactique en avançant des éléments dont on peut apprécier l'éventuelle pertinence à la lumière des analyses a priori épistémologique et didactique faites plus haut. Avec la méthodologie respectée ces deux dernières années, on peut observer qu'environ trois quarts des étudiants (une quarantaine sur les deux années) entrent dans cette dernière perspective sans que l'on ait pu départager le public des étudiants ayant choisi des études de mathématiques ou ceux qui ont une formation d'ingénieurs. Nous commentons ces résultats ci-après.

Comme dit plus haut, les problèmes mathématiques analysés dans cet article ont servi à alimenter des travaux proposés à nos étudiants dans une perspective d'initiation à l'analyse didactique. De 2003 à 2007, les modalités de travail étaient sensiblement 
différentes, les élèves-professeurs étant invités à construire par eux-mêmes un cours à partir de ces mêmes problèmes. Les observables récoltés alors ont été analysés par Rouy (2007) qui tire une conclusion assez négative :

« À quelques exceptions près, les élèves-professeurs ne parviennent pas à rentrer dans le jeu prévu. De plus, leur volonté d'exploiter le projet (soit pour des arguments relevant d'idéologies, soit parce que c'était notre consigne...) va les amener à développer des pratiques caractéristiques de l'ostension déguisée en essayant d'associer directement les éléments de théorie souhaités à des constats ne pouvant rester que visuels».

Nous pouvons voir, dans ces travaux, apparaître plusieurs phénomènes : une difficulté des futurs professeurs à appréhender le milieu de l'élève, la tendance à projeter dans les stratégies prévues des éléments standards de l'enseignement qui ne sont pas forcément appropriés, l'absence d'un discours technologique et la difficulté d'articuler mathématique et physique. Dans le nouveau dispositif, expérimenté avec Job, Rosseel et Schneider de 2009 à 2011, les résultats sont plus encourageants, plusieurs étudiants rentrant assez facilement dans une analyse épistémologique et didactique des problèmes concernés, ainsi qu'en témoignent leurs arguments. En même temps, il convient de rester modeste : il faut reconnaître que les questions posées lors du test à blanc rejoignaient, pour la plupart, celles qui leur avaient été proposées trois semaines auparavant pour guider leur lecture du projet et étayer leur réflexion. Qui plus est, le temps imparti leur laissait la possibilité de préparer l'épreuve en groupes et, ce n'est pas le moins bien sûr, un projet d'enseignement déjà construit leur était proposé. Il n'empêche que les étudiants devaient, lors du test à blanc, répondre de manière individuelle, et que leurs réponses respectives n'étaient pas spécialement copies conformes les unes des autres. En outre, et c'est là sans doute un résultat significatif de cette recherche bien qu'il ne soit guère possible de parler de réalité statistique sur une cohorte d'une quarantaine d'étudiants, leur réussite à ce test a eu une valeur prédictive dans l'évaluation de leur capacité d'analyse didactique en fin de formation, d'une part, et de la réflexivité dont ils ont pu faire preuve à propos des stages par rapport à une transposition didactique existante, d'autre part. Il est impossible de rendre compte ici de ces données en raison du fait qu'il faudrait alors rentrer dans l'analyse didactique des situations rencontrées et de la transposition dans laquelle elles s'inscrivent. Mais, de ce point de vue, la question 5 du test a été assez révélatrice car elle n'avait pas son équivalent dans la liste des questions préalables.

Il nous semble qu'a joué là un changement des modalités du dispositif de formation qui, sur base de mêmes problèmes mathématiques, s'est avéré pertinent. Tout reposait ici, non sur la construction d'un cours que les étudiants devaient s'imaginer dispenser eux-mêmes, mais sur l'analyse intellectuelle de variables didactiques de questions dévolues aux élèves. En outre, ce transfert était "sous contrat » au sein même d'une classe de situations didactiques d'une même catégorie, au sens où Schneider (2006b) le définit à propos de la résolution de problèmes mathématiques: l'existence de similitudes et de différences était annoncée d'entrée de jeu entre le problème du vase conique et celui des particules et le travail consistait à les identifier. Partant, les réactions des élèves au premier problème, qui avaient été analysées lors de la formation, permettaient d'anticiper l'impact du second problème sur les apprentissages. Sachant qu'il y avait possibilité de transfert au niveau de l'analyse didactique, restait donc aux étudiants à en préciser les tenants et les aboutissants, les deux problèmes étant suffisamment différents pour rendre non obvie la tâche proposée, ainsi que le montre l'analyse faite plus haut.

\section{EN GUISE DE CONCLUSION : LA PORTÉE DE CETTE RECHERCHE ET SES LIMITES}

Les données recueillies prennent sens au carrefour de deux problématiques auxquelles nos travaux antérieurs déjà cités apportent un éclairage particulier. D'une part, celle des obstacles d'apprentissage en mathématiques dont les élèves sont d'autant plus tributaires que ces obstacles ne sont pas pris en compte dans l'enseignement. Il s'agit ici de l'obstacle empiriste qui entrave particulièrement les apprentissages liés à l'analyse mathématique au niveau du lycée. Lequel obstacle est aggravé par les pratiques ostensives d'enseignement et la difficulté des professeurs, novices ou non, à concevoir des parcours qui les prennent en compte. D'autre part, la problématique des réformes éducatives, en particulier celle liée au paradigme des compétences et à sa promotion, 
auprès des acteurs de terrain, sur un mode essentiellement idéologique sur fond de théories socioconstructivistes sorties de leur contexte d'origine.

Au sein de la première problématique, nous avons spécifié une forme particulière de ce que Cirade (2006) appelle « les mathématiques comme problème professionnel ", plus propre aux futurs professeurs de lycée, en articulant fortement les connaissances disciplinaires et pédagogiques du PCK de Schulman (1987) par un questionnement épistémologique et didactique du savoir et de sa transposition. Cette forme se traduit en termes de praxéologies «modélisation » supposées prendre en compte les questions que les élèves se posent sur la modélisation mathématique de grandeurs qu'ils appréhendent de prime abord selon un filtre empiriste. L'absence d'identification de ce type de praxéologies et, a fortiori, la difficulté à les construire constituent alors une pierre d'achoppement du double forgetting qui suppose, en particulier, d'oublier les mathématiques de l'université avant de "retourner à l'école».

Quant à la seconde problématique, elle nous a engagés à concevoir une formation où l'arrière-fond socio-constructiviste ambiant pourrait devenir un levier pour lutter contre le monumentaliste dont souffre l'enseignement des mathématiques au prix toutefois d'un regard plus intellectualisé. Les théories didactiques que sont la TSD et la TAD y sont enseignées comme réseaux conceptuels permettant d'étudier des conditions sine qua non d'un enseignement inspiré de théories socio-constructivistes dans l'espoir de faire des formés de «potentiels vecteurs de changement ». Dans les travaux pratiques associés, ces derniers doivent pouvoir exploiter ces théories pour analyser des "pièces rapportées » de leurs stages ou du terrain et des ingénieries didactiques issues de la recherche. L'enjeu de formation, pour eux, est alors double : d'une part, dépasser une posture trop exclusivement idéologique vis-à-vis des réformes éducatives pour se polariser sur une analyse didactique de situations d'enseignement et, d'autre part, ouvrir leur conscience à un champ plus vaste de possibles.

C'est dans un tel contexte de formation que nous avons, en tant que chercheurs, délimité une question de recherche à portée essentiellement méthodologique : quels sont les indicateurs qui permettent de différencier les étudiants qui rencontrent le premier de ces enjeux par rapport aux autres étudiants ? La réponse construite ici montre que l'on ne peut se contenter d'indicateurs de surface, par exemple, le ressenti des étudiants, ni même le seul fait qu'ils aient choisi un dispositif d'enseignement faisant la part belle à l'activité de l'élève. Les indicateurs sont ici, au contraire, à déterminer sur base d'un double niveau d'analyses a priori. D'abord, celles qui portent sur le matériau qu'on fait travailler aux étudiants : les potentialités de dévolution des situations didactiques en termes de caractère fondamental et de milieu adidactique induit par leurs variables didactiques, les obstacles d'apprentissages, les formes embryonnaires des savoirs et les éventuelles transpositions didactiques lacunaires ou caduques. Ensuite seulement et, à la lumière de ce premier niveau, des analyses relatives à la manière dont les élèves-professeurs s'emparent des spécificités didactiques du même matériau pour mener une réflexion qui leur est propre : ici, sur la comparaison de deux ingénieries. Ces indicateurs ne peuvent donc être génériques car ils sont spécifiques d'une ingénierie ou d'une situation didactique donnée.

On peut voir aussi le choix du matériau comme faisant partie de la méthodologie : ici, un thème d'analyse mathématique à propos duquel nous avons construit des ingénieries calibrées et validées, reposant sur des praxéologies « modélisation » à propos desquelles notre formation gère de nombreux savoirs épistémologiques et didactiques. Il y a donc a priori matière à transférer de la part des formés. En effet, les pièces rapportées du terrain (manuels scolaires, documents d'accompagnement des programmes, notes d'enseignants...) sont, elles, assez pauvres et vite invalidées, en raison principalement de l'absence de caractère fondamental eu égard au savoir visé, ce qui court-circuite toute réflexion sur leurs variables didactiques (voir, sur ce point Schneider, 2008). Au-delà de cette visée méthodologique, le travail sur les ingénieries a aussi pour fonction, comme décrit plus haut, d'élargir le champ de conscience des formés afin de leur faire percevoir d'autres transpositions didactiques mais cet objectif-là ne fait pas l'objet premier de notre propos.

Comme développé plus haut, nous avons pu observer des transferts de savoirs enseignés dans la formation chez les trois quarts des étudiants d'une cohorte d'une quarantaine dont tous louent, ne fût-ce que par contrat, les vertus d'un enseignement faisant la part belle à l'activité de l'élève. Et ce, dans des conditions particulières qui sont alors des variables didactiques du dispositif de formation lui- 
même : le transfert était ici lui aussi « sous contrat », c'est-à-dire annoncé comme possible et, qui plus est, à l'intérieur d'une même catégorie de situations didactiques. Cela dit, nous avons remarqué que ces étudiants ont été les meilleurs pour mener, lors de cette formation, d'autres analyses didactiques ce qui relevait là de transferts plus glorieux. Nous ne pouvons cependant pas en tirer là matière à validation de notre recherche : les circonstances au cours desquelles les étudiants ont fait des analyses didactiques ayant été trop diverses de l'un à l'autre, déjà pour en rendre compte ici et, a fortiori, en tirer des généralités. Nous n'avons guère non plus de feedback des formés devenus professeurs. Mais plusieurs d'entre eux reviennent en formation continuée avec des questions dont l'acuité didactique nous paraît significative d'une progression. La plupart du temps, ils reviennent plusieurs années après la formation, le temps sans doute de s'intégrer dans la profession et de régler des questions de plus grande urgence comme celles liées à la discipline. Les autres étudiants, on les perd de vue...

Il n'empêche que, dans le cadre de cette formation et plus spécialement grâce à sa portée phénoménotechnique, notre expérimentation et nos analyses supra permettent de mettre en évidence des types d'observables qui caractérisent une aptitude requise par le métier d'enseignant : savoir analyser des situations didactiques, à la lumière de cadres théoriques et sans a priori idéologique. Cette aptitude des élèvesprofesseurs à rentrer dans l'analyse proprement didactique n'est en rien une condition suffisante pour devenir de bons enseignants. Mais elle nous apparaît comme une condition sine qua non d'une future pertinence avec laquelle ils choisiront ou concevront des dispositifs didactiques dans l'exercice de leur profession. Encore faut-il qu'ils le veuillent et que leurs conditions de travail le leur permettent. Nous y reviendrons.

Cette aptitude telle que caractérisée dans notre recherche en rejoint une autre identifiée par Bloch (2009), soit « la capacité du professeur à renvoyer aux élèves des réactions mathématiquement pertinentes ", au-delà de « conceptions très formelles des mathématiques ». Notre entreprise diffère cependant de celle de cette chercheuse par deux aspects au moins. Premièrement, nous avons modélisé un aspect de ce qu'on peut appeler une conception formelle des mathématiques, à savoir, dans les exemples qui nous concernent, une difficulté à concevoir des praxéo- logies «modélisation » en évitant des praxéologies « déduction » caduques au sens où l'entend Rouy (2007), c'est-à-dire amputées de maillons de la chaîne déductive. Qui plus est, cette modélisation fait partie intégrante du savoir didactique enseigné aux professeurs stagiaires, en souhaitant ainsi les rendre conscients et critiques de l'enjeu majeur au principe de la construction des ingénieries qu'on leur fait analyser.

Deuxièmement, a contrario du travail de Bloch, notre analyse ne porte pas sur les échanges, en classe, des professeurs stagiaires avec leurs élèves. On en reste donc ici au niveau du professeur « noosphérien $»$. Or plusieurs chercheurs insistent, dans leurs travaux, sur les interactions du professeur avec ses élèves lors du déroulement du cours car ils étudient la pratique enseignante de manière holistique comme un système cohérent et complexe : en plus des chercheurs déjà cités, signalons que c'est là le point de vue de Robert et Rogalski (2002) ainsi que celui de Margolinas et al. (2005). C'est aussi le nôtre. Il nous a paru cependant pertinent d'aménager et d'étudier des moments d'étude lors de la formation initiale d'élèves-professeurs où ceux-ci ne sont pas trop contraints ni par les injonctions institutionnelles qui pèsent sur le fonctionnement des institutions scolaires, ni par les obligations que leur font les maîtres de stages dont certains vont jusqu'à imposer leurs propres notes de cours. Car, de l'observation d'élèves-professeurs en classe, il n'est pas facile de distinguer ce qui relève du fonctionnement institutionnel ou de leurs propres aptitudes : en effet, on peut difficilement analyser leurs interactions avec les élèves sans les rapporter aux transpositions ou dispositifs en vigueur dans lesquels elles s'inscrivent. D'où notre idée de les observer au travail sur des ingénieries validées afin de tester leur aptitude à rentrer dans les modes de validation des chercheurs et, de là aussi, le double niveau d'analyses illustré plus haut, nos observables sur les élèves-professeurs ne pouvant être interprétés qu'à la lumière de l'analyse didactique a priori des situations didactiques qu'on soumet à leur sagacité.

En définitive, notre expérimentation s'inscrit donc dans une stratégie de formation qui se situe en amont de l'action dans l'idée « d'armer l'observation de l'acteur », « d'alerter l'environnement cognitif » afin d'éviter une "imprégnation socialisante trop exclusivement marquée par la culture locale » et de favoriser une « confrontation au champ réel de l'acti- 
vité sur un mode réflexif plutôt « qu'imprégnatif » (Perrenoud, 1994 ; Le Boterf, 2000 ; Beckers, 2007). Elle ne dispense évidemment pas d'une réflexion complémentaire "sur » et " dans » l'action et au travail de « debriefing en aval de l'action » (Pastré, 2002). Elle ne prétend pas non plus couvrir tous les paramètres qui interféreront dans les choix didactiques que fera le futur professeur au sein de sa pratique professionnelle. Par ailleurs, vu l'objet de cette recherche, il serait intéressant de la croiser avec d'autres recherches sur les résistances des professeurs, novices ou chevronnés, à adopter des ingénieries issues de la recherche, que ces résistances soient transversales, par exemple relatives à leur conception des mathématiques, ou spécifiques des contenus à enseigner. Mais il y a lieu aussi de s'interroger sur les caractéristiques des professeurs capables de s'emparer de telles ingénieries, en particulier sur les routines dont on sait l'importance dans la pratique professionnelle mais qui seraient celles de professeurs « dévoluant » ou « noosphériens »...

\section{NOTES}

1. Le paradigme socio-constructiviste renvoie ici à une idéologie d'enseignement qui fonctionne comme « valeur» prônée par la noosphère, particulièrement comme discours justifiant des enseignements organisés selon ce qu'on appelle communément l'APC ou Approche Par Compétences. Nous y reviendrons.

2. Le fait de rencontrer d'autres façons de faire et de penser que les nôtres nous fait prendre conscience que nos habitudes de pensée ne sont pas un donné inéluctable mais un construit possible.

\section{RÉFÉRENCES}

Artigue, M. (1990). Ingénierie didactique. Recherches en didactique des mathématiques, 9(3), 281-307.

Artigue, M. (2007). La didactique des mathématiques face aux défis de l'enseignement des mathématiques. -Exposé fait au Colloquium de didactique des mathématiques. Paris.

Artaud, M., \& Cirade, G. (2008). Lenseignement et la réception de la notion de PER dans la formation des professeurs stagiaires de mathématiques. Actes $d u$ Colloque international "Efficacité et Équité en Éducation », 32-46. Université Rennes 2. Repéré à [http:// esup.espe-bretagne.fr/efficacite_et_equite_en_education/programme/symposium_chevallard.pdf]

Ball, D. L., Thames, M. H., \& Phelps, G. (2008). Content knowledge for teaching: What makes it special? Journal of Teacher Education, 59(5), 389-407.

Beckers, J. (2007). Compétences et identité professionnelles : l'enseignement et autres métiers de l'interaction humaine. Bruxelles : De Boeck.

Bloch, I. (1999). Larticulation du travail mathématique du professeur et de l'élève dans l'enseignement de l'analyse en première scientifique. Recherches en Didactique des Mathématiques, 19(2), 135-193.

Bloch, I. (2009). Les interactions mathématiques entre professeurs et élèves. Comment travailler leur pertinence en formation ? Petit $x, 81,25-53$.

Bourdieu, P., Chamboredon, J.-C., \& Passeron, J.-C. (1983). Le métier de sociologue (4e édition). Paris : Mouton.

Brousseau, G. (1998). Théorie des situations didactiques. Grenoble : La Pensée Sauvage.

Chevallard, Y. (1991), La transposition didactique : du savoir savant au savoir enseigné. Grenoble: La Pensée Sauvage.

Chevallard, Y. (1992). Concepts fondamentaux de la didactique : perspectives apportées par une approche anthropologique. Recherches en Didactique des Mathématiques, 12(1), 72-112.

Chevallard, Y. (1999). Lanalyse des pratiques enseignantes en théorie anthropologique du didactique. Recherches en Didactique des Mathématiques, 19(2), 221-265.

Chevallard, Y. (2000). Enseignement insensé, enseignement raisonné et créativité sociale. Communication au colloque Mathématiques sans frontières 2000. IUFM d'Aix-Marseille.

Chevallard, Y. (2007). Un concept en émergence : la dialectique des médias et des milieux. Communication au Séminaire national de didactique des mathématiques 2007. Paris, 23 mars 2007.

Cirade, G. (2006). Devenir professeur de mathématiques : entre problèmes de la profession et formation en IUFM. Les mathématiques comme problème professionnel (Thèse de doctorat, université de Provence-Marseille).

Clivaz, S. (2014). Des mathématiques pour enseigner. Grenoble: La Pensée Sauvage. 
Cornu, B. (1983). Apprentissage de la notion de limite: conceptions et obstacles (Thèse de doctorat, université de Grenoble).

Crahay, M., \& Forget, A. (2006). Changements curriculaires: quelle est l'influence de l'économique et du politique ? Dans F. Audigier, M. Crahay, J. Dolz \& A. Delhaxhe (dir.), Curriculum, enseignement et pilotage. Bruxelles: De Boeck.

Freudenthal (1973). Mathematics as an educational task. Dordrecht : D. Reidel.

Gantois, J. Y., \& Schneider, M. (2012). Un milieu graphico-cinématique pour l'élaboration d'une technologie des dérivées dans une praxéologie «modélisation ». Recherches en Didactique des Mathématiques, 32(1), 57-99.

Ginzburg, C. (2001). A distance. Paris : Gallimard.

Houdement, C., \& Kuzniak, A. (1996). Autour des stratégies utilisées pour former les maîtres du premier degré en mathématiques. Recherches en Didactique des Mathématiques, 16(3), 289-322.

Job, P. (2011). Étude du rapport à la notion de définition comme obstacle à l'acquisition du caractère lakatosien de la notion de limite par la méthodologie des situations fondamentales / adidactiques (Thèse de doctorat, université de Liège).

Job, P., \& Schneider, M. (2011). Une situation fondamentale du concept de limite ? Question de langage, de culture ? Comment la TAD permet-elle de problématiser cette question ? Communication au $2^{\mathrm{e}}$ congrès Théorie Anthropologique du Didactique. Uzès, novembre 2007.

Job, P., \& Schneider, M. (2014). Empirical positivism, an epistemological obstacle in the learning of calculus. ZDM The international Journal on Mathematics Education, 46(4), 635-646. Heidelberg : Springer.

Lalande, A. (2002). Dictionnaire de philosophie. Paris : Presses universitaires de France.

Le Boterf, G. (2000). De quel concept de compétences les entreprises et les administrations ont-elles besoin ? Dans C. Bosman, F.-M. Gerard \& X. Roegiers (dir.), Quel avenir pour les compétences? (p. 15-19). Bruxelles : De Boeck.

Ma, L. (1999). Knowing and Teaching Elementary Mathematics: Teachers' Understanding of Fundamental Mathematics in China and the United States. London : Lawrence Erlbaum Associates Publishers.

Margolinas, C. (2004). Points de vue de l'élève et du professeur. Essai de développement de la théorie des situations didactiques. France : université de Provence. Repéré à [https://tel.archives-ouvertes.fr/tel-00429580/fr/]

Margolinas, C., Coulange, L., \& Bessot, A. (2005). What can the teacher learn in the classroom. Educational Studies in Mathematics, 59, 205-234.

Pastré, P. (2002). Lanalyse du travail en didactique professionnelle. Revue française de Pédagogie, 138, 9-17.

Perrenoud, Ph. (1994). La formation des enseignants : entre théorie et pratique. Paris : L'Harmattan.
Popper, K. (1973). La logique de la découverte scientifique. Paris : Payot.

Robert, A., \& Rogalski, J. (2002). Le système complexe et cohérent des pratiques des enseignants de mathématiques : une double approche. Revue Canadienne de l'Enseignement des Sciences, des Mathématiques et des Technologies, 2(4), 505-528.

Roegiers, X. (2000). Une pédagogie de l'intégration. Compétences et intégration des acquis dans l'enseignement. Bruxelles : De Boeck.

Rouy, E. (2007). Formation initiale des professeurs (du secondaire supérieur) et changements de posture vis-à-vis de la rationalité mathématique (Thèse de doctorat, université de Liège).

Rouy, E. (2009). Confrontation d'élèves-professeurs à une ingénierie sur les dérivées : ce qui ne (se) passe pas. Dans Y. Matheron \& S. René de Cotret (dir.), La désaffection envers l'étude des mathématiques: entre problématiques curriculaires et didactiques (p. 28-40). Repéré à [http:// fastef.ucad.sn/EMF2009/Projet\%20sp\%E9ciaux/Projet\%20special\%201/actes_projet1_final.pdf]

Salin, M.-H. (1999). Pratiques ostensives des enseignants. Dans G. Lemoyne \& F. Conne (dir.), Le cognitif en didactique des mathématiques (p. 327-352). Montréal : Les presses de l'université de Montréal.

Schneider, M. (1988). Des objets mentaux « aire " et « volume " au calcul des primitives (Thèse de doctorat, Université catholique de Louvain).

Schneider, M. (1991a). Un obstacle épistémologique soulevé par des « découpages infinis » des surfaces et des solides. Recherches en Didactique des Mathématiques, 11(2.3), 241-294.

Schneider, M. (1991b). Quelques difficultés d'apprentissage du concept de tangente. Repères IREM, 5, 65-82.

Schneider, M. (1992). À propos de l'apprentissage du taux de variation instantané. Educational Studies in Mathematics, 23, 317-350.

Schneider, M. (2001). Praxéologies didactiques et praxéologies mathématiques. À propos d'un enseignement des limites au secondaire. Recherches en Didactique des Mathématiques, 21(1.2), 7-50.

Schneider, M. (2006a). Quand le courant pédagogique « des compétences » empêche une structuration des enseignements autour de l'étude et de la classification de questions parentes. Revue Française de Pédagogie, 154, 85-96.

Schneider, M. (2006b). Comment des théories didactiques permettent-elles de penser le transfert en mathématiques ou dans d'autres disciplines ? Recherches en Didactique des Mathématiques, 26(1), 9-38.

Schneider, M. (2007). Les compétences comme cadre pour organiser des enseignements de mathématiques? Oui, mais... Quelques dérives possibles. Canadian Journal of Science, Mathematics and Technology Education, 7(1), 28-40.

Schneider, M. (2008). Traité de Didactique des Mathématiques. Liège : Presses universitaires de Liège. 
Schneider, M. (2011a). Ingénieries didactiques et situations fondamentales. Quel niveau praxéologique ? Dans C. Margolinas, M. Abboud-Blanchard, L. Bueno-Ravel, N. Douek, A. Fluckiger \& P. Gibel (dir.), En amont et en aval des ingénieries didactiques (p. 175-206). Grenoble : La Pensée Sauvage.

Schneider, M. (2011b). Mise en œuvre de l'approche par compétences en Communauté française de Belgique : ce que la recherche en didactique des mathématiques aurait pu ou pourrait apporter. Dans J. Lebeaume, A. Hasni \& I. Harlé (dir.), Recherches et expertises pour l'enseignement de la technologie, des sciences et des mathématiques. Louvain-la-Neuve : De Boeck.

Schneider, M. (sous presse). Utiliser les potentialités phénoménotechniques de la TAD : quel prix payer? Conférence plénière au $4^{\mathrm{e}}$ Colloque sur la Théorie Anthropologique du Didactique. Toulouse, avril 2013. À paraître dans les Actes.

Shulman, L. S. (1987). Knowledge and teaching: Foundations of the new reform. Harvard Educational Review, Feb. 1987, 1-22.

Sierpinska, A. (1985). Obstacles épistémologiques relatifs à la notion de limite. Recherches en Didactique des Mathématiques, 6(1), 5-68.

Silvy, C., Delcroix, A., \& Mercier, A. (2013). Enquête sur la notion de "pedagogical content knowledge », interrogée à partir du « site local d'une question ». Éducation E Didactique, 7(1), 33-57. 